Gdańsk 2020, Nr. 43

https://doi.org/10.26881/sgg.2020.43.07

\title{
Ružena Kozmová
}

Universität Trnava (Slowakei) / Univerzita sv. Cyrila a Metoda, Trnava

https://orcid.org./0000-003-3606-4391

\section{Zur linguistischen Forschung in der slowakischen Germanistik. Eine kommentierte Bibliographie}

Der Beitrag setzt sich zum Ziel, die linguistische Forschung in der Slowakei zu skizzieren. Dabei wird auf Aufsätze hingewiesen, die innerhalb der letzten zehn Jahre entstanden sind. Diese Bestandsaufnahme knüpft an Berichte über die linguistische Forschung der ersten zwanzig Jahre nach der Wende an. Die Forschungslage, die sich gewissermaßen konstituiert hat, lässt sich von zwei Standpunkten aus beobachten. Zum einen ist es die Forschung der Institute, die ununterbrochen (vor und nach der Wende) fortgesetzt wird, zum anderen ist es die Forschung an den neu etablierten Instituten bzw. Lehrstühlen der deutschen Sprache und Literatur.

Schlüsselwörter: Germanistische Linguistik, Forschung, Übersetzen, Korpuslinguistik, Kollokationsforschung, Pragmatik, Semantik

Linguistic Research in Slovak German Studies. A Commented Bibliography. - The aim of this article is to outline the linguistic research in Slovakia which consists of papers and scientific studies published by Slovak scholars of German Studies over the last ten years. At the same time, this paper will follow up on the report on linguistic research in Slovakia within twenty years after the political change. The research situation has stabilized to some extent and can be characterised in two respects. Firstly, it is the research conducted continuously at the departments before and after the political change. Secondly, it is the research on newly established institutes respectively on departments of German language and literature.

Keywords: German Linguistics, Translation, Corpus Linguistics, Collocations, Pragmalinguistik, Semantics

Im Beitrag wird die Forschungslage der Germanistik in der Slowakei analysiert, wobei auf die Arbeiten der letzten zehn Jahre hingewiesen wird. Der Beitrag bemüht sich, eine freie Fortsetzung der Analyse der vergangenen zwanzig Jahre ${ }^{1}$ zu sein; dadurch wird an die ersten Forschungsergebnisse der germanistischen Linguistik in der Slowakei angeknüpft. Ausgespart wird eine nähere Charakteristik der einzelnen germanistischen Institute bzw. Lehrstühle der

1 Die Bestandsaufnahme über die Germanistik in der Slowakei im Zeitraum 1990-2010 ist erfasst in der Slowakischen Zeitschrift für Germanistik, Jg. 3, Heft 2, 2011. 
deutschen Sprache und Literatur hinsichtlich der Studentenanzahl, der Besetzung der einzelnen Arbeitsstellen, der durch den Verband der Deutschlehrer organisierten Fachtagungen, etc. Die Publikationen von Durčo (2017a) und Zemaníková (2017) erfassen die Situation in der Germanistik an den slowakischen Universitäten im letzten Jahrzehnt.

Die Germanistik wird hier im Sinne der germanistischen Linguistik verstanden, es geht uns mehr um die Forschungsprofilierung der einzelnen Institute, die in einige Richtungen zielt. Zum einen sind es die ältesten Institute, die die klassische philologische Ausbildung (vor allem die Geschichte der deutschen Sprache, die Übersetzungswissenschaft) anbieten, zum anderen sind es Institute, die bestrebt sind, moderne linguistische Disziplinen wie Korpuslinguistik, Konstruktionsgrammatik, Semantik und Pragmatik systematisch zu erforschen. Des Weiteren ist auch die Forschung im Bereich Lexikologie, Phraseologie, Lexikografie, lexikale Semantik und Textlinguistik zu nennen. Schließlich lässt sich noch die auf die extremistischen Tendenzen innerhalb Europas reagierende Forschung von Schuppener $(2010,2017)$ hinzufügen.

Die Forschungslage in der Slowakei ist sehr heterogen: Zum einen ist es die Forschung auf dem Gebiet der Geschichte der deutschen Sprache, die ununterbrochen, europäische Grenzen überschreitend, vor und nach der politischen Wende erforscht wird und von einer erfolgreichen internationalen Zusammenarbeit zeugt. Es handelt sich insbesondere um die Untersuchung der Relikte der deutschen Mundarten in der Zips von Papsonová (2011, 2012a, 2012b, 2013, 2014, 2015a, 2015b, 2016), in der Mittel- und Westslowakei von Piirainen (2004), Meier / Ziegler (2001) und Ziegler (2003). An diese Forschung knüpften die Arbeiten der jüngeren slowakischen Kolleginnen in Bratislava (Mayerová 2009, 2013, 2014), Nitra (Kretterová 2008) und Banská Bystrica (Bohušová 2017a) an. In diesem Zusammenhang ist die aktuelle internationale Forschung der tschechischen (Philosophische Fakultät der Karlsuniversität ${ }^{2}$ ) und slowakischen Kollegen (Philosophische Fakultät der Universität der Heiligen Cyril und Method in Trnava ${ }^{3}$ ) zu nennen, die Mundartenrelikte der Waldleute (Hundshäcker) und deren Lebensweise untersuchen. Thematisch lässt sich darunter die letzte Arbeit von Tancer (2016) einordnen, die in Zusammenarbeit mit dem Karpathendeutschen Verband entstanden ist.

Eines der ältesten germanistischen Institute: das Institut für Germanistik, Nederlandistik und Skandinavistik der Comenius Universität in Bratislava mit dem Studienangebot für Übersetzer, Dolmetscher und künftige Deutschlehrer forscht insbesondere auf dem Gebiet der Übersetzungswissenschaft. Gleich nach der Wende entstand eine große Nachfrage nach Übersetzern und Dolmetschern im Bereich der Rechtssprache, deren Kenntnis von den europäischen Institutionen verlangt wurde. Die Übersetzungswissenschaft, das Streben nach neuen Methoden und Ansätzen wird zum Forschungsthema auch anderer germanistischen Institute

2 Šatava, L. ist Koordinator des internationalen Projekts „Rozmery revitalizácie etnickej minority na Slovensku a v Čechách: Interdisciplinárny záchranný výskum zanikajúcej etnickej skupiny Huncokárov“ [Dimensionen der Revitalisierung der ethnischen Minorität in der Slowakei und in Böhmen: Eine interdisziplinäre Rettungsforschung der aussterbenden ethnischen Hundshäckergruppe, Übers.: R.K.]. Im Rahmen dieses Projekts wird die Sprache der Kolonisten, der Waldleute (Hundshäcker), ihre Lebensweise, ihre Mundart in Böhmen und in der Westslowakei (die Gegend von Modra, Pezinok, Limbach) erforscht.

3 Am Projekt beteiligen sich mehrere Institute der Philosophischen Fakultät der UCM Trnava, insbesondere das Institut für Ethnologie (Nováková Slobodová u. a.) und das Institut für Germanistik (Schuppener u. a.). 
(Banská Bystrica, Nitra, Prešov). Zu erwähnen ist die Arbeit von Bohušová (2017b), in der sie die Theorie der translatologischen Neutralisationen verfasste und definierte.

In der Rechtsspracheforschung entstanden viele Aufsätze, die der Analyse allgemeiner Fragen des Übersetzens von Rechtstexten sowie der Aufgaben des Gerichtsgutachters und des Gerichtsübersetzers gewidmet sind. Mit dem Dolmetschen und Übersetzen aus der Sicht der Gesetzgebung der Slowakei bzw. der EU setzen sich Ďuricová (2009a, 2009b, 2011), Lauková (2013), Štefková (2013) und Gergel (2014, 2016, 2017a, 2017b) auseinander. Guldanová untersucht die Tätigkeit des Dolmetschers und Übersetzers in der Gerichtspraxis (2013a, 2013b, 2014). Fachsprachenforschung, besonders im Bereich der Wirtschaftssprache, wurde an der Fakultät für angewandte Sprachen der Wirtschaftsuniversität in Bratislava betrieben (Ondrčková / Lišková 2011).

Sisák (2011) spielte eine führende Rolle in der Forschung zur Textlinguistik am Institut für Germanistik in Prešov. Die Untersuchungen von Sisák mit dem Schwerpunkt Wortschatz und Wortbildung konzentrierten sich auf die prozessuelle und die analytische Wortbildung, wobei die Frage der Nomination durch Wortbildung im Vordergrund stand. Eine systematische Erforschung von Textlinguistik im Allgemeinen, teilweise an Fachsprachen (insbesondere der Rechtssprache) orientiert, stellen die Arbeiten von Vajičková dar. Ihre Studien knüpfen frei an die Arbeiten von Sisák (Wortbildung und lexikalische Semantik) ${ }^{4}$ und diejenigen von Mlacek (1977) (Phraseologie) an. Vajičková widmete sich den einzelnen Phänomenen der lexikalischen Semantik und der Stilistik. Es sei dabei nur auf einige Themen und Themenbereiche verwiesen, wie der Stil im Allgemeinen (2011), Wiederholung und Variation (2009).

Im Zusammenhang mit der neuesten Forschung ist die fruchtbare nationale Forschungsaktivität im Bereich der Kollokationsdidaktik (Ďurčo / Vajíčková et al. 2017, 2018, 2019a, 2019b, 2019c), Duurčo / Vajičková / Tomášková (2019) sowie die internationale Forschung im Bereich der Phraseologie und Sprichwörterkunde zu nennen (Durčo 2012, 2013, Durčo / Steyer / Hein 2015).

Einen Aufschwung in der Korpuslinguistik - besonders in der Kollokationsforschung, in der kontrastiven Lexikographie, in der Konstruktionsgrammatik und Pragmatik - bemerkt man seit Jahren am Germanistischen Institut der Philosophischen Fakultät der Universität der Heiligen Cyril und Method in Trnava. Die Forschung konzentriert sich um Peter Durčo, der den Versuch unternommen hat, die slowakische kontrastive Lexikographie (2011) zu systematisieren. Seine größte Aufmerksamkeit schenkte er der durch viele wissenschaftliche Projekte unterstützten Korpuslinguistik (Ďurčo 2010a, 2010b) und Kollokationsforschung. Er legte die Grundlage für ein Kollokationsmodell (eine Kollokationsmatrix), formulierte die Methodologie und die Testverfahren für die Erfassung und Beschreibung von Kollokationen; gemeinsam mit seinen Doktoranden und Assistenten erarbeitete er kontrastive Kollokationsmodelle von Adjektiven, Verben und Substantiven (Ďurčo / Banášová / Hanzlíčková 2010). Die Kollokationsforschung am Germanistischen Institut in Trnava mündete in eine fruchtbare internationale Zusammenarbeit mit dem Institut für Deutsche Sprache in Mannheim $^{5}$ sowie mit dem Germanistischen Institut in Santiago de Compostela. Diese fruchtbare

4 Eine ausführliche Bibliographie von Sisák ist der Slowakischen Zeitschrift für die Germanistik, Jg. 3, H. 2 (2011) zu entnehmen.

5 Hervorzuheben ist hier die schon seit Jahren bestehende erfolgreiche Zusammenarbeit mit Kathrin Steyer. 
Kooperation führte zu zahlreichen Publikationen zur Erforschung von minimalen usuellen Wortverbindungen und Wortverbindungsmustern (Ďurčo 2018a, 2018b, Ďurčo 2019, Ďurčo et al 2019, Ďurčo / Tabačeková (Hg.) 2019, Fraštíková 2018, Hein et al. 2018, Hornáček Banášová 2018a, 2018b, 2018c, Tabačeková 2017, 2018, 2019a, 2019b, 2019c).

Mit der Kollokationsforschung von Verben hängt frei die Erforschung der Verbvalenz (Kozmová 2013a, 2013b, Duurčo et al. 2014, Fraštíková 2014, Dujková / Hornáček Banášová 2016) zusammen. Kozmová sieht die Möglichkeit der Weiterentwicklung der Valenztheorie in der interaktiven Einbeziehung der Umgebung der verbalen Valenzträger. In ihren Arbeiten werden die Valenzpartner als gleichberechtigte Glieder angesehen, und der Aufbau des Satzes ergibt sich aus der Zusammenführung semantischer Komplexe. Die Valenz als komplexes Phänomen wird im Bereich der Semantik und Pragmatik weiter präzisiert, insbesondere in den Aufsätzen von Kozmová / Miháliková (2018).

Die Kollokationsforschung aus didaktischer Sicht wird am Lehrstuhl für deutsche Sprache und Literatur der Pädagogischen Fakultät in Bratislava betrieben (Ďurčo 2015, 2016, 2017b Ďurčo / Vajičková et al. 2017, Duurčo / Vajičková, Tomášková 2019, Ďurčo / Vajičková et al. 2018, 2019a, 2019b, Vajičková / Durčo et al. 2017, Vajičková / Durčo et al. 2018).

In den letzten Jahren erschienen auch Publikationen zu pragmalinguistischen Aspekten der gesprochenen Sprache und ihrer Relevanz für den Fremdsprachenunterricht (Tomášková 2017, 2018, 2019a, 2019b, Vajičková / Tomášková 2014).

Zusammenfassend lässt sich Folgendes feststellen: In den drei Jahrzehnten, die seit der politischen Wende inzwischen vergangen sind, hat sich in der Forschung der slowakischen Germanistik viel Positives ereignet. Es kam nicht nur zur Stabilisierung der Fachkräfte, sondern auch zu systematischen Untersuchungen im Bereich der germanistischen Linguistik. Es handelt sich insbesondere um die Studien zur Korpuslinguistik, Kollokationsforschung, Phraseologie, Textlinguistik, Semantik und Pragmalinguistik. Nennenswert ist auch die sich dynamisch entwickelnde Forschung im Bereich der Didaktik, wobei die Kollokationsdidaktik als vorherrschend gilt.

\section{Literatur}

Bohušová, Zuzana (2017a): Nárečie L'upoče: O dialekte severozápadného Novohradu [Die Mundart von L'upoča: Von dem Dialekt des nordwestlichen Novohrad]. Banská Bystrica: Univerzita Mateja Bela.

Bohušová, Zuzana (2017b): Sprachlich und kommunikativ bedingte Neutralisierungen beim Dolmetschen. Wien: Praesens.

Dujková, Simona / Hornáček Banášová, Monika (2016): Vergleich der lexikografischen Beschreibung des Verbs erfahren mit dessen Gebrauch in den Kollokationen im Hinblick auf die Vermittlung der Problematik für die FS-Lerner / 2016. In: Ďurčo, Peter (Hg.): Kollokationsforschung und Kollokationsdidaktik. Wien: LIT, 11-39.

Durčo, Peter (2010a): Einsatz von SketchEngine im Korpus - Vorteile und Mängel. In: Ptashnyk, Stefaniya / Hallsteinsdóttir, Erla / Bubenhofer, Noah (Hg.): Korpora, Web und Datenbanken: computergestützte Methoden in der modernen Phraseologie und Lexikographie. Baltmannsweiler: Schneider-Verl. Hohengehren, 119-131. 
Durčo, Peter (2010b): Extracting Data from Corpora Statistically - Pros and Cons. In: Ďurčo, Peter (Hg.): Feste Wortverbindungen und Lexikographie. Kolloquium zur Lexikographie und Wörterbuchforschung. Berlin / New York: De Gruyter, 43-50.

Durčo, Peter (2011): Deutsch-slowakische Lexikografie: Allgemeine D-S/S-D-Wörterbücher vor und nach der Wende. In: Slowakische Zeitschrift für Germanistik. Jg. 3, H. 2, 66-75.

Duurčo, Peter (2012): Diasystematische Differenzen von Sprichwörtern aus der Sicht der kontrastiven Parömiografie. In: Steyer, Kathrin (Hg.): Sprichwörter multilingual. Theoretische, empirische und angewandte Aspekte der modernen Parömiologie. Tübingen: Narr, 357-377.

Durčo, Peter (2013): Extensionale und intensionale Äquivalenz in der Phraseologie am Beispiel von deutschen und slowakischen Sprichwörtern. In: Benayoun, Jean-Michel / Kübler, Natalie / Zouogbo, Jean-Philippe (Hg.): Parémiologie. Proverbs et formes voisines. Tome 2, Presses Universitaires de Sainte Gemme, 49-64.

Ďurčo, Peter (2015): Gebrauch der Kollokationen und Probleme der zweisprachigen Kollokationslexikografie für Lerner. In: Schmidlin, Regula / Behrens, Heike / Bickel, Hans (Hg.): Sprachgebrauch und Sprachbewusstsein. Implikationen für die Sprachtheorie. Berlin / Boston: De Gruyter, 221-237.

Durčo, Peter (2016): Zum Konzept der Kollokationsdidaktik und des Kollokationslernens bei Germanistikstudenten. In: Durčo, Peter (Hg.): Kollokationsforschung und Kollokationsdidaktik. Wien: LIT, 147-172.

Ďurčo, Peter (2017a): Deutsche Sprache und Germanistik an den slowakischen Universitäten in Zahlen. In: Goltschnigg, Dietmar (Hg.): Wege des Deutschen. Deutsche Sprache und Germanistik-Studium aus internationaler Sicht. Tübingen: Stauffenburg, 175-180.

Durčo, Peter (2017b): Korpuslinguistik als Innovation im DaF-Studium und Korpora im DaF-Unterricht. Fallbeispiel Trnava (Slowakei). In: Jesenšek, Vida (Hg.): Germanistik in Maribor. Tradition und Perspektiven. Maribor: Univerza v Mariboru, 33-38.

Ďurčo, Peter (2018a): Faktoren der konvergenten und divergenten Äquivalenz von präpositionalen Wortverbindungen: Deutsch-Slowakisch. In: Steyer, Kathrin (Hg.): Sprachliche Verfestigung. Wortverbindungen, Muster, Phrasem-Konstruktionen. Tübingen: Narr, 285-306.

Ďurčo, Peter (2018b): Lexikalisierte PWVs aus kontrastiver Sicht. In: Hornáček Banášová, Monika / Fraštíková, Simona (Hg.): Aktuelle Fragen und Trends der Forschung in der slowakischen Germanistik. Nümbrecht: Kirsch-Verlag, 9-59.

Duurčo, Peter (2019): Präpositionale Wortverbindungen im Kontrast Deutsch-Slowakisch. Bestandsaufnahme über die Schwerpunkte und Zwischenbilanz der Ergebnisse. In: Ďurčo, Peter / Tabačeková, Jana (Hg.): Präposition-Nomen-Verbindungen. Korpusstudien zu Gebrauch und Musterhaftigkeit phraseologischer Minimaleinheiten. Berlin: Logos, 178-191.

Durčo, Peter / Banášová, Monika / Hanzlíčková, Astrid (2010): Feste Wortverbindungen im Kontrast. Trnava: Univerzita sv. Cyrila a Metoda.

Duurčo, Peter et al. (2014): Valenz und Kookkurrenz. Grammatische und lexikologische Ansätze. Berlin: LIT.

Durčo, Peter / Steyer, Kathrin / Hein, Katrin (2017): Sprichwörter im Gebrauch. Mannheim: Institut für Deutsche Sprache.

Durčo, Peter / Vajičková Mária et al. (2017): Kollokationen im Unterricht. Ein Lehr-und Übungsbuch. Nümbrecht: Kirsch-Verlag.

Ďurčo, Peter / Vajičková, Mária et al. (2019a): Kollokationen I. Ein Übungsbuch. Nümbrecht: KirschVerlag. 
Durčo, Peter / Vajičková, Mária et al. (2019b): Kollokationen III. Ein Übungsbuch. Nümbrecht: Kirsch-Verlag. Durčo, Peter / Vajičková, Mária / Tomášková, Simona (2019): Kollokationen im Sprachsystem und Sprachgebrauch. Ein Lehrbuch. Nümbrecht: Kirsch-Verlag.

Durčo, Peter / Hornáček Banášová, Monika / Fraštíková, Simona / Tabačeková, Jana (2019): Zur Äquivalenz der minimalen lexikalisch geprägten Muster „Präposition+Substantiv“ im deutschslowakischen Kontrast. In: Yearbook of Phraseology. Vol. 10, No. 1-2, 141-172.

Durčo, Peter / Tabačeková, Jana (Hg.) (2019): Präposition-Nomen-Verbindungen. Korpusstudien zu Gebrauch und Musterhaftigkeit phraseologischer Minimaleinheiten. Berlin: Logos.

Durčo, Peter / Tabačeková, Jana (in Druck): Das phraseologische Modell des Typs Präposition + Substantiv und Relevanz der Gebrauchsaspektenanalyse aus kontrastiver Sicht. In: Mellado Blanco, Carmen (Hg.): Formulaic Language: A Multilingual Approach to the Phraseological Patterns. Berlin u. a.

Duricová, Alena (2009a): Einige Bemerkungen zum Genitivgebrauch im Deutschen und Slowakischen. In: Helin, Irmeli (Hg.): Linguistik und Übersetzung in Kuovola. Beiträge zu Sprache und Sprachen 7. Helsinki: Yliopistopaino, 216-229.

Ďuricová, Alena (2009b): Überlegungen zum Gebrauch und zur Übersetzung von Eigenamen in den Gerichtstexten. In: LINGUA VIVA. Pedagogická fakulta Jihočeské univerzity: České Budějovice. Jg. V, Nr. 9, 49-55.

Ďuricová, Alena (2011): Untersuchungen zu deutschen Fachsprachen in der Slowakei. In: Slowakische Zeitschrift für Germanistik. Jg. 3, H. 2, 76-84.

Fraštíková, Simona (2014): Das Valenz-Kollokationssyntagma konverser Verben „mieten“ und „vermieten“. In: Slowakische Zeitschrift für Germanistik. Jg. 6, Nr. 2, 46-64.

Fraštíková, Simona (2018): Die korpusbasierte Untersuchung der „lokalen“ Präposition-Substantiv-Verbindung am Telefon aus kontrastiver Sicht. In: Hornáček Banášová, Monika / Fraštíková, Simona (Hg.): Aktuelle Fragen und Trends der Forschung in der slowakischen Germanistik 3. Nümbrecht: Kirsch-Verlag, 60-106.

Gergel, Peter (2014): Auf Deutsch verfasste Tyrnauer Bürgertestamente der ersten Hälfte des 19. Jahrhunderts. In: Vajičková, Mária et al. (Hg.): Aufdem Weg zu Texten und Kontexten: Festschrift für Ivan Cvrkal. Nümbrecht: Kirsch-Verlag, 149-178.

Gergel, Peter (2016): Ekvivalencia v nemeckej, slovenskej, českej a mad'arskej trestnoprávnej terminológii na príklade foriem trestnej činnosti [ ̈̈quivalenz in der deutschen, tschechischen und ungarischen strafrechtlichen Terminologie am Beispiel der Formen von strafbaren Handlungen]. In: Filologické štúdie 2. Nümbrecht: Kirsch-Verlag, 35-57.

Gergel, Peter (2017a): Slovenská právna terminológia v trnavských meštianskych závetoch prvej polovice 19. storočia [Slovakische Rechtsterminologie in den Tirnauer städtischen Testamenten aus der ersten Hälfte des 19. Jh.s]. In: Slovenská reč. Jg. 82, Nr. 3, 249-255.

Gergel, Peter (2017b): Ekvivalenčná analýza věeobecných trestnoprávnych pojmov [Äquivalenzanalyse allgemeiner strafrechtlicher Begriffe]. Nümbrecht: Kirsch-Verlag.

Guldanová, Zuzana (2013a): Der Gerichtsdolmetscher/Gerichtsübersetzer und die Skopostheorie (am Beispiel des slowakischen Gesetzes über Sachverständige, Dolmetscher und Übersetzer). In: Lachout, Martin (Hg.): Aktuelle Tendenzen der Sprachwissenschaft. Hamburg: Dr. Kovač, $127-132$.

Guldanová, Zuzana (2013b): Problém preložitel'nosti / nepreložitel'nosti v kontexte súdneho tlmočenia / prekladu [Das Problem der Übersetzbarkeit / Nichtübersetzbarkeit im Kontext des 
Gerichtsdolmetschens / Gerichtsübersetzens]. In: Guldanová, Zuzana (Hg.): Kontexty súdneho prekladu a tlmočenia II. Bratislava: Univerzita Komenského Bratislava, 51-60.

Guldanová, Zuzana (2014): Niekol'ko pohladov na činnost súdneho tlmočníka / prekladatel'a zo súdnej praxe [Einige Einblicke in die Tätigkeit des Gerichtsdolmetschers / Gerichtsübersetzers in der Gerichtspraxis]. In: Guldanová, Zuzana (Hg.): Kontexty súdneho prekladu a tlmočenia III. Bratislava: Univerzita Komenského Bratislava, 50-67.

Hein, Katrin / Ďurčo, Peter / Mellado Blanco, Carmen / Steyer, Kathrin (2018): Am Anfang - na začiatku - al principio. Eine musterbasierte Fallstudie im Sprachvergleich. In: Steyer, Kathrin (Hg.): Sprachliche Verfestigung. Wortverbindungen, Muster, Phrasem-Konstruktionen. Tübingen: Narr, 307-339.

Hornáček Banášová, Monika (2018a): Kollokationsforschung und Kollokationen in den Bildungsstandards. In: Janíková, Věra et al. (Hg.): Sprachen verbinden. Beiträge der 24. Linguistik- und Literaturtage. Brno, Tschechien 2016. Hamburg: Dr. Kovač, 231-240.

Hornáček Banášová, Monika (2018b): Präpositionale Wortverbindungen mit modaler Bedeutung. In: Hornáček Banášová, Monika / Fraštíková, Simona (Hg.): Aktuelle Fragen und Trends der Forschung in der slowakischen Germanistik 3. Nümbrecht: Kirsch-Verlag, 147-171.

Hornáček Banášová, Monika (2018c): Präpositionale Wortverbindungen mit modaler Bedeutung. In: Monika Hornáček Banášová / Simona Fraštíková (Hg.): Aktuelle Fragen und Trends der Forschung in der slowakischen Germanistik 3. Nümbrecht: Kirsch-Verlag, 147-171.

Kozmová, Ružena (2011): DaF-Grammatik in der Slowakei. In: Slowakische Zeitschrift für Germanistik. Jg. 3, H. 2, 9-27.

Kozmová, Ružena (2013a): Theoretische Ausgangsbasis eines kontrastiven Verbvalenzmodells. In: Slowakische Zeitschrift für Germanistik. Jg. 5, H. 2, 5-27.

Kozmová, Ružena (2013b): Von der Funktion zur Bedeutung. Verbvalenz kontrastiv. Berlin: LIT.

Kozmová, Ružena / Miháliková, Lucia (2016): Valenz und Kollokationen als Ausdruck der Interaktion von Grammatikalisierungs- und Lexikalisierungsprozessen. Eine Analyse am Beispiel der präfigierten be-Verben. In: Ďurčo, Peter (Hg.): Kollokationsforschung und Kollokationsdidaktik. Wien: LIT, 88-108.

Kozmová, Ružena / Miháliková, Lucia (2018): Polysemie des Verbs als Problem der Semantik-PragmatikSchnittstelle. In: Germanica Wratislaviensia 143. Acta Universitatis Wratislaviensis No. 3835, 227-244.

Kretterová, Ludmila (2008): Mestská kniha Banskej Štiavnice (1486-1575): fonematicko-grafematická analyza [Das Stadtbuch von Banská Štiavnica (1486-1575): eine phonematisch-graphematische Analyse]. Nitra: UKF.

Lauková, Jana (2013): Sprachen im Vergleich. Syntaktische Koordination und Subordination in der deutschen und slowakischen Sprache. Ludwigsburg: Pop.

Mayerová, Erika (2009): Zur Sprache der Pressburger Zünfte des 16. Jahrhunderts. In: Deutsche Sprache in der Slowakei. Bratislava: Metodicko-pedagogické Zentrum, 287-292.

Mayerová, Erika (2013): Jazyk banského práva v prameni „Eysen-Hämmer Ordnungen/poriadok pre železiarske hámre (v stoliciach Abov, Spiš, Gemer a Turňa 1713-1715)“. In: Kontexty súdneho prekladu a tlmočenia 2, Bratislava: Univerzita Komenského, 61-69.

Mayerová, Erika (2014): Aus dem Stadtbuch von Schmöllnitz / Smolník. In: Kontexty súdneho prekladu a tlmočenia 3. Bratislava: Univerzita Komenského, 78-87. 
Meier, Jörg / Ziegler, Arne (Hg.) (2001): Deutsche Sprache in Europa: Geschichte und Gegenwart; Festschrift für Ilpo Tapani Piirainen zum 60. Geburtstag. Wien: Praesens.

Mlacek, Jozef (1977): Slovenská frazeológia [Slowakische Phraseologie]. Bratislava: Slovenské pedagogické nakladatel'stvo.

Ondrčková, Eva / Lišková, Danuša (2011): Thema Wirtschaft in den deutsch-slowakischen lexikografischen Werken. In: Slowakische Zeitschrift für Germanistik. Jg. 3, H. 2, 85-94.

Papsonová, Mária (2011): Die Sprachgeschichts- und Mundartforschung in den beiden letzten Jahrzehnten. In: Slowakische Zeitschrift für Germanistik. Jg. 3, H. 2, 28-42.

Papsonová, Mária (2012a): Kanzleisprachenforschung und Rechtsgeschichte. In: Greule, Albrecht / Meier, Jörg / Ziegler, Arne (Hg.): Kanzleisprachenforschung. Ein internationales Handbuch. Berlin / Boston: De Gruyter, 53-67.

Papsonová, Mária (2012b): Die deutsche Kanzleisprache in der Slowakei. In: Greule, Albrecht / Meier, Jörg / Ziegler, Arne (Hg.): Kanzleisprachenforschung. Ein internationales Handbuch. Berlin / Boston: De Gruyter, 557-569.

Papsonová, Mária (2013): Einige Bemerkungen zur diachronen Sprachbetrachtung in der Auslandsgermanistik. In: Meier, Jörg / Puchalová, Ingrid (Hg.): Perspektiven der Auslandsgermanistik. Berlin: Weidler Buchverlag, 75-84.

Papsonová, Mária (2014): Sasko-Magdeburské právo na Slovensku. Krajinské právo v Žilinskej knihe [Sächsisch-Magdeburgisches Recht in der Slowakei. Das Landrecht im Silleiner Buch]. Bratislava: Eurokódex.

Papsonová, Mária (2015a): Freud und Leid des Übersetzens von historischen Texten. In: Bachmann, Armin R. / Simet, Katrin (Hg.): Zwischen Donau, Hornad und Dunajetz. Zur deutschen Sprache in der Slowakei. Berlin: Weidler Buchverlag, 77-93.

Papsonová, Mária (2015b): Eberhard Windecke und seine Denkwürdigkeiten zur Geschichte des Zeitalters Kaiser Sigismunds. In: Greule, Albrecht / Rössler, Paul unter Mitarbeit von Katrin Simet (Hg.): Sprachbrücken. Aktuelle Forschungen zur deutschen Sprache und Literatur in der Slowakei. Berlin: Weidler Buchverlag, 111-121.

Papsonová, Mária (2015c): Kahlenberg - Kalenberk - Gol'embark - Lysá nad Dunajcom. Zur Herkunft und Entwicklung von geografischen Namen der Zips. In: Drechsler-Meel, Heike / Schmitt, Heinz (Hg.): Karpaten Jabrbuch 2016 (= Kalender der Karpatendeutschen aus der Slowakei). Stuttgart: Arbeitsgemeinschaft der Karpatendeutschen aus der Slowakei, 133-145.

Papsonová, Mária (2016): K niektorým právno-historickým a sociolingvistickým aspektom prekladu nemeckej právnej zbierky v Žilinskej knihe [Zu einigen rechtshistorischen und soziolinguistischen Aspekten der Übersetzung der Rechtssammlung im Silleinbuch]. In: Historické štúdie 50. Bratislava: Veda, 21-33.

Piirainen, Ilpo Tapani (Hg.) (2004): Deutsche Sprache in der Slowakei II. Geschichte, Gegenwart und Didaktik. Wien: Praesens.

Schuppener, Georg (2010): Sprache des Rechtsextremismus. Spezifika der Sprache rechtsextremistischer Publikationen und rechter Musik. Leipzig: Edition Hamouda.

Schuppener, Georg (2017): Sprache und germanischer Mythos im Rechtsextremismus. Leipzig: Edition Hamouda.

Sisák, Ladislav (2011): Semantik und Wortbildung in Forschung und Lehre in der Slowakei (1990-2011). In: Slowakische Zeitschrift für Germanistik. Jg. 3, H. 2, 44-54. 
Štefková, Markéta (2013): Právny text v preklade: translatologické aspekty právnej komunikácie v kombináciách málo rozširených jazykov [Rechtstext in der Übersetzung: translatologische Aspekte der Rechtskommunikation in der Kombination mit den wenig verbreiteten Sprachen]. Bratislava: Iura edition.

Tabačeková, Jana (2017): Formale Abgrenzung der präpositionalen Wortverbindungen. In: Vajičková, Mária et al. (Hg.): Filologické stúdie. Nümbrecht: Kirsch-Verlag, 14-20.

Tabačeková, Jana (2018): Die präpositionale Wortverbindung „im Handumdrehen“. Eine kontrastive Studie. In: Hornáček Banášová, Monika / Fraštíková, Simona (Hg.): Aktuelle Fragen und Trends der Forschung in der slowakischen Germanistik III. Nümbrecht: Kirsch-Verlag, 107-146.

Tabačeková, Jana (2019a): Die Problematik der Äquivalenz der präpositionalen Wortverbindungen als phraseologischen Einheiten. In: Kvapil, Roman et al. (Hg.): Cudzie jazyky v premenách času 9 $=$ Foreign languages in changing times 9. Bratislava: Ekonóm, 96-108.

Tabačeková, Jana (2019b): Präpositionale Wortverbindungen. Eine kontrastive Fallstudie. Berlin: Logos.

Tabačeková, Jana (2019c): Die PWV „na počkanie“. Eine Fallstudie. In: Ďurčo, Peter / Tabačeková, Jana (Hg.): Präposition-Nomen-Verbindungen. Korpusstudien zu Gebrauch und Musterhaftigkeit phraseologischer Minimaleinheiten. Berlin: Logos, 105-114.

Tancer, Jozef (2016): Rozviazané jazyky. Ako sme hovorili v starej Bratislava [Die gelösten Zungen. So haben wir in Alt-Bratislava gesprochen]. Bratislava: Slovart.

Tomášková, Simona (2017): Pragmatische Aspekte der Verwendung von Formeln in mündlicher Interaktion. In: Filologické śtúdie 3. Nümbrecht: Kirsch-Verlag, 31-39.

Tomášková, Simona (2018): Pragmatische Aspekte des Gesprächs. Nümbrecht: Kirsch-Verlag.

Tomášková, Simona (2019a): Pragmatische Aspekte der Kollokationen in mündlicher Kommunikation. In: Duurčo, Peter / Vajičková, Mária / Tomášková, Simona: Kollokationen im Sprachsystem und Sprachgebrauch: Ein Lehrbuch. Nümbrecht: Kirsch-Verlag, 163-190.

Tomášková, Simona (2019b): Ausgewählte gesprächsanalytische Aspekte und ihre Relevanz für den DaF-Unterricht. In: Barras, Malgorzata et al. (Hg.): IDT 2017, Bd. 2: Sektionen: Brücken gestalten - mit Deutsch verbinden: Menschen - Lebenswelten - Kulturen. Beiträge der 16. Internationalen Tagung der Deutschlehrerinnen und Deutschlehrer. Berlin: Schmidt, 273-279.

Vajičková, Mária (2009): Wiederholung und Variation als stilistische Prinzipien in belletristischen und wissenschaftlichen Texten. In: Contemporary views on theory and practice of foreign language teaching 2. Praha: Kernberg Publishing, 107-117.

Vajičková, Mária (2011): Zur slowakischen germanistischen Phraseologie und Stilistik nach der Wende. In: Slowakische Zeitschrift für Germanistik. Jg. 3, H. 2, 29-43.

Vajičková, Mária / Ďurčo, Peter (2017): Didaktische Aspekte der Kollokationen. In: Ďurčo, Peter /Vajičková, Mária et al.: Kollokationen im Unterricht: Ein Lehr-und Übungsbuch. Nümbrecht: Kirsch-Verlag, 119-144.

Vajičková, Mária / Ďurčo, Peter et al. (2018): Kollokationen II. Ein Übungsbuch. Nümbrecht: Kirsch-Verlag. Vajičková, Mária / Tomášková, Simona (2014): Zum Gespräch unter linguistischen und didaktischen Aspekten. In: Studies in Foreign Language Education 6. Nümbrecht: Kirsch-Verlag, 138-150.

Zemaníková, Nadežda (2017): Germanistik in der Slowakei. In: Mitteilungen des deutschen Germanistenverbandes. H. 1, 106-110.

Ziegler, Arne (2003): Aufgaben und Desiderata der germanistischen Sprachgeschichtsforschung und der universitären Ausbildung in der Slowakei. In: Greule, Albrecht / Meier, Jörg (Hg.): Deutsche Sprache in der Slowakei. Bilanz und Perspektiven ihrer Erforschung. Wien: Praesens, 55-68. 